\title{
Impact of Raising Switching Stages on the Reliability of Interconnection Networks
}

\author{
Fathollah Bistouni $^{1}$, Mohsen Jahanshahi ${ }^{2, *}$ \\ ${ }^{1}$ Department of Computer Engineering, Central Tehran Branch, Islamic Azad University, Tehran, Iran. \\ Email: fat.bistouni.eng@iauctb.ac.ir \\ ${ }^{2}$ Department of Computer Engineering, Central Tehran Branch, Islamic Azad University, Tehran, Iran. \\ IEEE Senior Member, Email: mjahanshahi@iauctb.ac.ir \\ *Corresponding author
}

How to cite this paper: Fathollah Bistouni, Mohsen Jahanshahi (2020). Impact of Raising Switching Stages on the Reliability of Interconnection Networks. Journal of the Institute of Electronics and Computer, 2, 93-120.

https://doi.org/10.33969/JIEC.2020.21007.

Received: March 19, 2020

Accepted: April 3, 2020

Published: April 6, 2020

Copyright $(92020$ by author(s) and Institute of Electronics and Computer. This work is licensed under the Creative Commons Attribution International License (CC BY 4.0).

http://creativecommons.org/licenses/by/4.0/

\section{(c) (i) Open Access}

\begin{abstract}
Multistage Interconnection Networks (MINs) are a cost-effective idea for connection between different nodes in multi-processor systems. In the meantime, understanding the impact of increasing the number of stages (i.e. a famous method for improving fault tolerance) on the reliability of these networks is essential. Last studies on this challenging issue, revealed that adding one switching stage to MINs is preferable to two in terms of reliability. However, there were some non-negligible gaps in previous researches: (i) The probability of failure for terminal nodes was not considered in the calculation of reliability. (ii) Numerical results were only based on the small size of the network, i.e. $8 \times 8$. (iii) Reliability analyses were time-independent. (iv) The lack of some important parameters such as the mean time to failure and availability was noticeable. Therefore, this paper will take a more comprehensive analysis of reliability to meet the above specifications.
\end{abstract}

\section{Keywords}

Shuffle-exchange networks, Reliability, Fault tolerance, Mean time to failure, Failure rate, Availability

\section{Introduction}

Interconnection networks (INs) are responsible to interconnect processors, memories and other peripherals within parallel computers, which require some kinds of communication subsystems to do so. Moreover, most of the proposals in the context of ATM (Asynchronous Transfer Mode) networks follow self-routing MINs (Multistage Interconnection Networks) [1, 2]. In addition, different proposed interconnection topologies for parallel computing have been examined and adapted to networks-on-chip (NoCs) [3, 4]. 
Performance and cost are two key parameters in selecting an appropriate IN. In addition, MINs are one of the cost-effective options to meet these needs [5-17]. On the other hand, reliability is an essential requirement for improving the efficiency of these networks [18-26].

One popular idea to improve the fault tolerance and reliability of the MINs is increasing the number of switching stages. Therefore, in order to evaluate the effect of increasing the number of stages on reliability, Ref. [27] examines the network reliability (i.e. all terminal reliability) of the most recognized MINs named shuffle-exchange network (SEN) and SEN+ (SEN with one additional stage). In this work, an exact reliability equation for the SEN of size $\mathrm{N} \times \mathrm{N}$ was obtained. Also, relatively accurate reliability equations were calculated for the SEN+ of size $8 \times 8$ and $16 \times 16$ using the continuous-time Markov chain (CTMC) approach. In addition, an approximation technique for determining the reliability of the larger SEN+s was considered by providing reliability bounds. In this study, it was shown that the SEN+ was superior to the SEN in terms of reliability. In other words, this research reflects the fact that adding one additional stage to the SEN can result in improved reliability. For a more comprehensive analysis, in Ref. [28], a reliability analysis of the SEN, SEN+, and SEN+2 (SEN with two additional stages) was conducted to assess the impact of several additional stages on the SEN reliability. In this study, it was concluded that the SEN+ had the highest reliability and SEN+2 had the lowest one. Also, SEN achieved higher reliability than SEN+2. This latter result was surprising, since in previous studies, SEN has been considered the most unreliable networks, and it failed in case of a single fault. Therefore, a re-analysis of the reliability of three networks of SEN, SEN+, and SEN+2 was performed in [29]. The results of this study indicated that $\mathrm{SEN}+$ and $\mathrm{SEN}+2$ held the same functionality in terms of reliability. Also, the reliability of these networks is always more than the single-path SEN reliability. Therefore, it was concluded that one extra stage could achieve better performance for SEN compared to two extra stages.

Although the previous works $[28,29]$ are able to provide useful information about the impact of increasing the number of stages on the reliability, there are some gaps in these works as follows: (i) Terminal nodes failure are not taken into account in the analysis of reliability. (ii) A small network size (i.e. $8 \times 8$ ) is used to obtain the numerical results. (iii) Reliability analyses were static in terms of time. (iv) Important metrics of mean time to failure (MTTF) and availability have not been evaluated, but these metrics are very effective to determine the efficiency of a system in terms of reliability.

In addition, it should be noted that although some of the reported works meet some of the requirements above they are not complete enough. For this purpose, in [19], 
Fathollah Bistouni et al.

terminal and broadcast reliability for SEN and SEN+ have been analyzed properly. However, this work does not fully assess the impact of increasing the number of stages on reliability for the following reasons: (1) It covers the two networks SEN and SEN+. Therefore, this study is only appropriate for investigating the impact of a single stage on the given MINs. However, we need to understand the impact of multiple stages in MINs as well. To achieve this goal, SEN+2 network should also be taken into account in the reliability analysis. (2) It covers just two aspects of reliability: terminal and broadcast reliability. However, a comprehensive analysis is required to analyze the network reliability too. (3) Although the probability of failure is intended for switch nodes, it is not considered for terminal nodes in the calculation of reliability. While, there is the possibility of failure for the terminal nodes such as processors and memory modules in a real system. (4) Availability parameter (most common system reliability metric used in repairable systems) is not analyzed. As another example, in [22], proper analysis has been done on the networks of SEN and SEN+ in terms of terminal reliability. However, there are also important gaps in this work similarly to that of the work in [19]. This means that for a comprehensive analysis, we also need to analyze the reliability of the SEN+2. In addition, all three aspects of reliability namely terminal reliability, broadcast reliability and network reliability need to be analyzed. Also, a reasonable assumption is that there is a possibility of failure in the terminal nodes like switch nodes.

Although full analyzes have been done in the area of MINs reliability, no one can provide a comprehensive picture of the impact of increasing the number of switching stages on these networks. However, increasing the number of stages in the MINs is a common technique to improve the fault tolerance and reliability of MINs. Therefore, a proper understanding of the impact of this technique on MINs reliability is crucial and valuable for researchers in this area. Consequently, the purpose of this paper is to undertake a more extensive analysis of reliability in order to meet the above needs and is also to obtain more comprehensive information about the MINs reliability.

The rest of this paper is organized as follows: SEN, SEN+, and SEN+2 network structures will be described in section 2. Time-independent and time-dependent reliability will be analyzed in sections 3 and 4, respectively. The MTTF will be evaluated in section 5. Availability will be analyzed in section 6. An analytical discussion will be given in section 7. At the end, some conclusions will be derived in section 8. 


\section{Background and contribution}

\subsection{The structure of networks}

In this sub-section, SEN, SEN+, and SEN+2 network structures will be explained. SENs of size $N \times N$ are comprised of $\left(\log _{2} N\right)$ stages, and each stage contains $N / 2$ switching elements of size $2 \times 2$. A SEN of size $8 \times 8$ is shown in Fig. 1 (a). The network complexity of an $\mathrm{N} \times \mathrm{N}$ SEN is $\frac{\mathrm{N}}{2}\left(\log _{2} \mathrm{~N}\right)$. In this network, there is only one path between each source-destination pair.

A SEN+ (SEN with one additional stage) of size $N \times N$ is comprised of $\left(\log _{2} N\right)+$ 1 stages, and each stage contains $\mathrm{N} / 2$ switching elements of size $2 \times 2$. A SEN + of size $8 \times 8$ is shown in Fig. 1 (b). The network complexity of an $\mathrm{N} \times \mathrm{N}$ SEN + network is $\frac{\mathrm{N}}{2}\left(\left(\log _{2} \mathrm{~N}\right)+1\right)$. In this network, there are two paths between each source-destination pair.

A SEN+2 (SEN with two additional stages) of size $\mathrm{N} \times \mathrm{N}$ is comprised of $\left(\log _{2} \mathrm{~N}\right)+2$ stages, and each stage contains $\mathrm{N} / 2$ switching elements of size $2 \times 2$. A SEN+2 of size $8 \times 8$ is shown in Fig. 1 (c). The network complexity of an $\mathrm{N} \times \mathrm{N}$ SEN+2 network is $\frac{N}{2}\left(\left(\log _{2} N\right)+2\right)$. In this network, there are four paths between each source-destination pair.

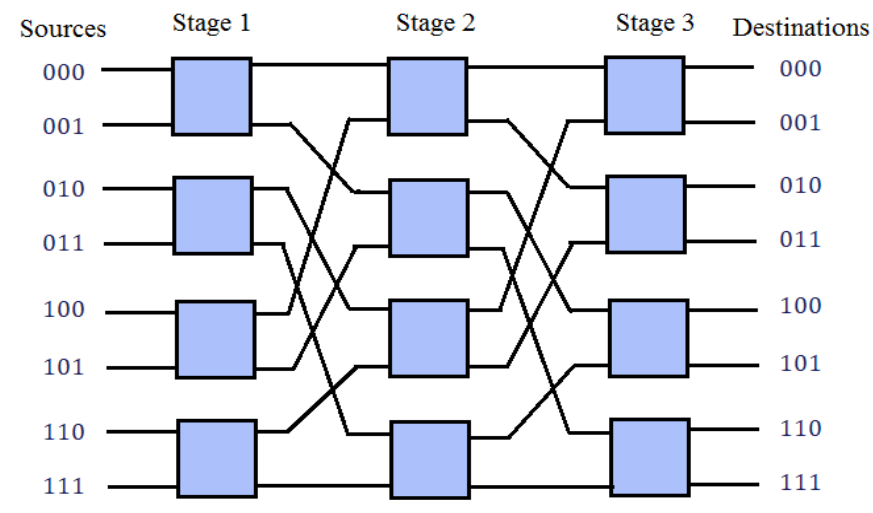

(a)

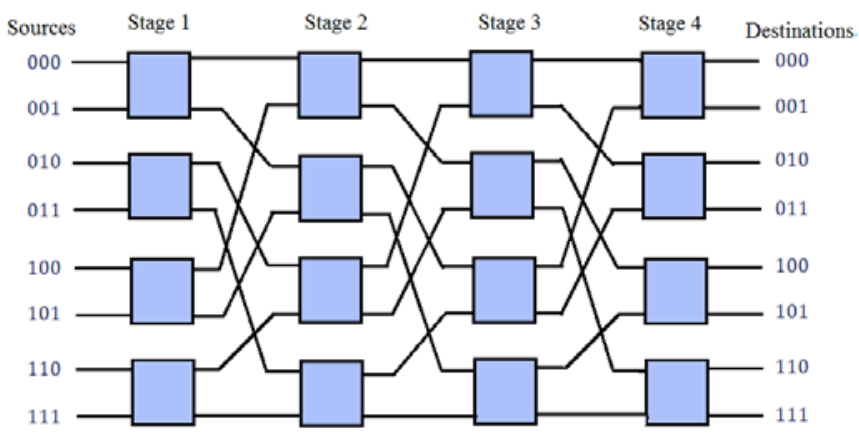

(b) 
Fathollah Bistouni et al.

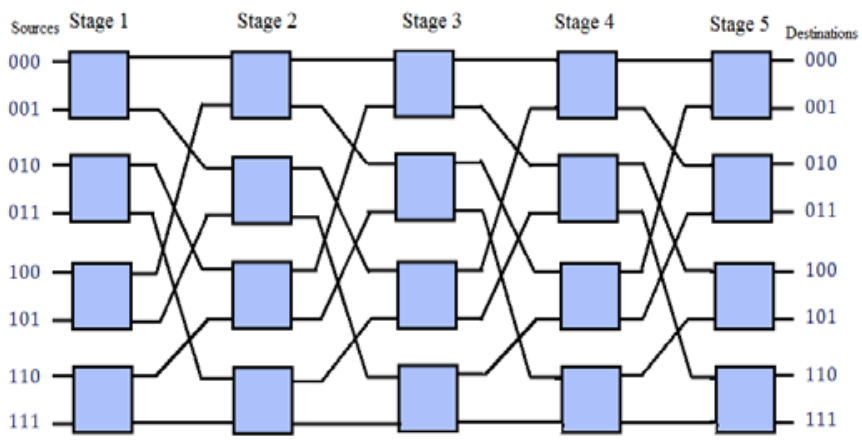

(c)

Figure 1. (a) A SEN of size $8 \times 8$, (b) A SEN + of size $8 \times 8$, and (c) A SEN+2 of size $8 \times 8$.

\subsection{Contribution}

This sub-section is set out to illustrate what are the contributions of this paper and what has been exploited from previous works.

Like Refs. [19, 22, 27, 28, 29], the first similarity of this research with previous works is the use of the same networks (i.e. SEN and its variants) for study. The reasons for this action are as follows: (1) The most important reason for this issue is to make this study comparable to previous works. Like many previous works, this research evaluates the SEN and its variants, thus the results of this research will be comparable to the results obtained from previous works. (2) SEN is topologically identical with many MINs known as Banyan-type MINs or single-path MINs such as Delta, Baseline, and Generalized Cube. Therefore, analyzes performed on the SEN will be applicable about the performance of all Banyan-type MINs.

In addition, it should be noted that in Section 3, Eqs. (1) through (9) are based on [29]. However, the analyzes made in Section 3 and all sections of the paper, as well as Eqs. (10) through (50) are part of the contributions of this paper.

Now, let us take a closer look at the contributions of this paper. The contributions for this paper can be listed as follows:

(1) Contributions in relation to analyze:

(a) In Section 3, an analysis of terminal reliability, broadcast reliability, and network reliability has been performed for SEN, SEN+, and SEN+2. In the previous works, the numerical results obtained for these three dimensions of reliability for the SEN and its variants were often based on a small network size of $8 \times 8$. However, the numerical analysis performed in Section 3 is for different network sizes from $8 \times 8$ through $2048 \times 2048$. (b) In the previous works, reliability analyzes conducted on the reliability of the SEN and its variants (i.e. SEN+ and SEN+2) were based on a time-independent strategy. In Section 4 of this paper, for the first time, a 
comprehensive analysis of the reliability of these networks is presented in all three reliability dimensions namely terminal, broadcast, and network as a function of time. In addition, different types of network size are considered in this analysis. Another noteworthy point in this analysis is that the probability of failure of memory modules and processors is also considered in the analysis of reliability, the assumption that was lost in previous works. (c) The time-to-failure analysis is a common analysis to assess reliability in terms of the expected time to failure. Many scholars in assessing the effectiveness of interconnection networks have also underlined it. However, none of the previous works has done this analysis on the SEN and its variants in order to evaluate the performance of these networks and thus the method of increasing the number of stages in terms of mean time to failure (MTTF). Therefore, in Section 5 of this paper, this parameter has been analyzed for different network sizes from $8 \times 8$ through $2048 \times 2048$. The reliability of processors and memory units are also taken into account in this analysis. (d) A common assumption is that the components of a system can be repaired in case of failure. In this case, the availability can be taken into account for the assessment of repairable systems. However, so far little attention has been paid to examining this metric in interconnection networks. In addition, to the best of our knowledge, the method of increasing the number of stages has not been investigated using SEN's availability analysis and its variants. That's why Section 6 of this article offers a detailed analysis of availability for SEN, SEN+, and SEN+2. In addition, this analysis has been done for different network sizes from $8 \times 8$ through $2048 \times 2048$.

According to the discussion above, important deficiencies of previous works have been solved in this paper. These cases can be summarized as follows: The different network sizes are considered in the time-independent analysis of reliability. The time-dependent reliability analysis is performed for the first time for all three networks of SEN, SEN+, and SEN+2, taking into account different network sizes and different reliability dimensions. In addition, the reliability of processors and memory modules is also considered in this paper. This case was not considered in almost any of the previous works in the area of MINs because of simplification in the calculations. Nevertheless, using this new methodology can improve considerably the accuracy of reliability analyzes. For the first time, MTTF analysis for all three SEN-based networks has been done in this paper taking into account different network sizes and different dimensions of reliability (i.e. terminal, broadcast, and network). The availability of which is one of the most important parameters in network reliability engineering has received little attention in the field of interconnection networks. Therefore, the other contribution of this paper id to analyze the availability of SEN, SEN+, and SEN+2, taking into account different 
Fathollah Bistouni et al.

network sizes.

As a result, it is quite evident that the analyzes carried out in this paper and the methodology used in these analyzes are quite comprehensive, accurate, and more reliable in comparison with previous works in this field.

(2) Contributions in relation to the results of this research:

In addition to the methodology used in the analyzes contained in this paper that solves many shortcomings in previous works, the results of these analyzes are very important as well. In order to clarify the significance of these results, first, the most important results of the previous works should be considered: In [27], in order to evaluate the efficiency of the method of increasing the number of stages, the reliability of the SEN and SEN+ is analyzed and compared with each other. According to this study, SEN+'s reliability is better than SEN's reliability, which results in the confirmation of the efficiency of increasing the number of stages in the MINs. In [28], a time-independent reliability analysis has been performed on the SEN, SEN+, and SEN+2 for a small network size of $8 \times 8$. According to this research, although SEN+ improves reliability compared to SEN, SEN+ reliability is the lowest reliability among these three networks. In other words, the study done in [28] confirmed the addition of one stage to the MINs, but it does not confirm adding more than one stage to them in terms of reliability. In [29], a more precise method has been used to analyze reliability in SEN, SEN+, and SEN+2 compared to the previous works. The results of this study showed that the SEN+ and SEN+2 are close in reliability, but their reliability are always better than reliability of the SEN. As can be seen, in previous works, using more precise methods, researchers have gradually achieved more accurate and new results about the efficiency of the method of increasing the number of stage in MINs. On the other hand, as discussed, one of the contributions of a paper is to provide the most complete reliability analysis using a more precise and comprehensive methodology compared to previous works. That is why in this paper, new results are obtained about the efficiency of the SEN and its variants (as will be analyzed in Sections 3 through 6), which modifies the results of previous studies. In summary, these results are as follows:

As will be analyzed in Sections 3 through 6, when the network size and operating time increases, SEN+2's reliability is higher than SEN and SEN+'s reliability. In other words, contrary to previous works, the results of this paper demonstrate that SEN+2 is a better option for use in large size and long working times. In addition, these results emphasize that the size of the system and the working time should be taken into account in choosing a suitable network. Nevertheless, these important metrics have been neglected in previous studies. 


\section{Time-independent reliability analysis}

Recently, in [29], in order to assess the impact of increasing the number of stages to the MINs, time-independent terminal, broadcast, and network equations were calculated for SEN, SEN+, and SEN+2. However, in this work, the numerical results were limited to a small network of size $8 \times 8$. Thus, to achieve a more comprehensive analysis, there is a need to analyze these networks in the scenario of large-scale networks. Therefore, the purpose of this section is to fill this gap.

Let $r$ be the probability of a switching element being operational. Based on [29], terminal reliability $\left(R_{T}\right)$, broadcast reliability $\left(R_{B}\right)$, and network reliability $\left(R_{N}\right)$ equations for SEN, SEN+, and SEN+2 of size $\mathrm{N} \times \mathrm{N}$ are given by:

$R_{T}(S E N)=r^{\log _{2} N}$

$R_{T}(S E N+)=r^{2}\left(1-\left(1-r^{\left(\log _{2} N\right)-1}\right)^{2}\right)$

$R_{T}(\operatorname{SEN}+2)=r^{2}\left(1-\left(1-\left(r^{2}\left(1-\left(1-r^{\left(\log _{2} N\right)-2}\right)^{2}\right)\right)\right)^{2}\right)$

$R_{B}(S E N)=r^{N-1}$

$R_{B}(S E N+)=r^{\frac{(N+2)}{2}}\left(1-\left(1-r^{\frac{(N-2)}{2}}\right)^{2}\right)$

$R_{B}(S E N+2)=\left(r^{\frac{(N+2)}{2}}\right)\left(1-\left(1-\left(r^{2}\left(1-\left(1-r^{\frac{(N-4)}{4}}\right)^{2}\right)\right)\right)^{2}\right)\left(\left(1-(1-r)^{2}\right)^{\frac{N-4}{4}}\right)$

$R_{N}(S E N)=r^{\frac{N}{2} \log _{2} N}$

$R_{N}(S E N+)=r^{N}\left(\left(1-(1-r)^{2}\right)^{\frac{N}{4}}\right)\left(1-\left(1-r^{\left.\frac{N}{4}\left(\log _{2} N\right)-2\right)}\right)^{2}\right)$

$R_{N}(S E N+2)=r^{N}\left(\left(1-(1-r)^{2}\right)^{\frac{N}{4}}\right)\left(1-\left(1-r^{\frac{N}{8}\left(\left(\log _{2} N\right)-2\right)}\right)^{4}\right)\left(1-\left(1-r^{\frac{N}{4}}\right)^{2}\right)$

According to the above equations, for a medium switch reliability $(r=0.95)$, terminal reliability, broadcast reliability, and network reliability analysis results in terms of network size are shown in Figs. 2, 3, and 4.

As previously mentioned, the results of [29] for network of size $8 \times 8$ showed that $\mathrm{SEN}+$ and SEN+2 had the same functionality in terms of reliability. Also, the reliability of these networks has been always more than the single-path SEN reliability. However, according to the Figs. 2, 3, and 4, it is obvious that as the 
Fathollah Bistouni et al.

network size increases, SEN+2 becomes more preferable to two other networks in terms of reliability. That is why the analysis of reliability is essential for large-scale network scenarios.

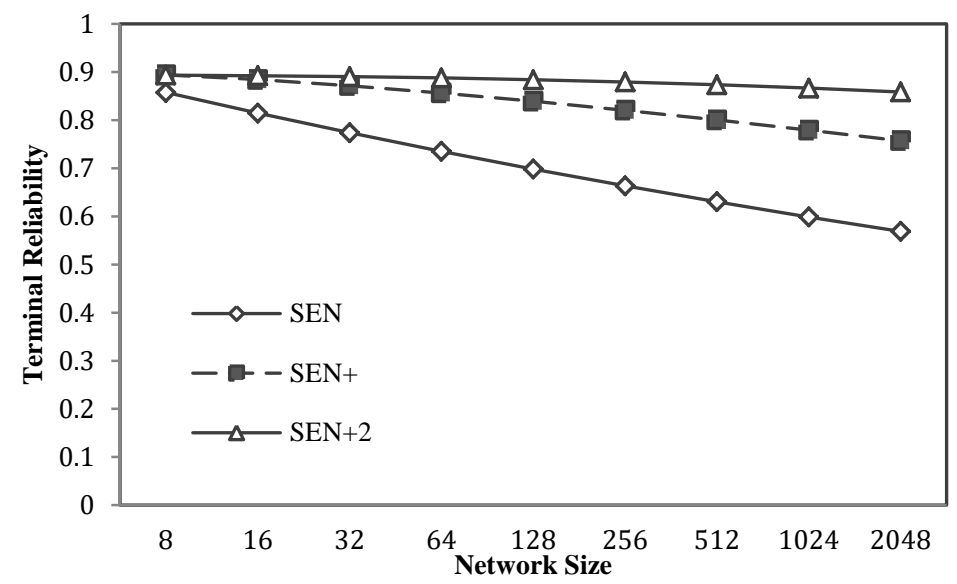

Figure 2. Time-independent terminal reliability vs. network size.

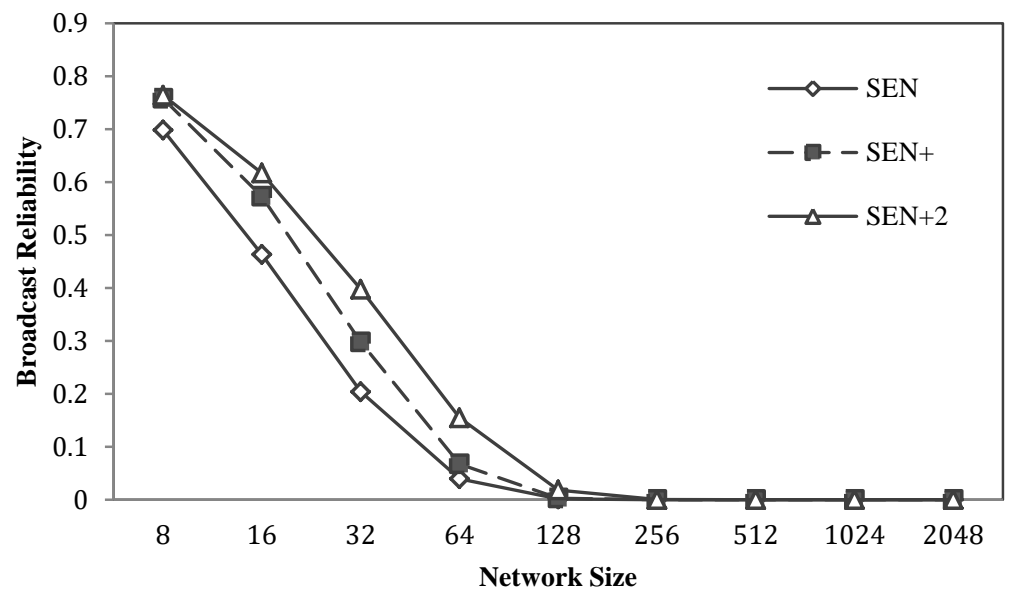

Figure 3. Time-independent broadcast reliability vs. network size. 


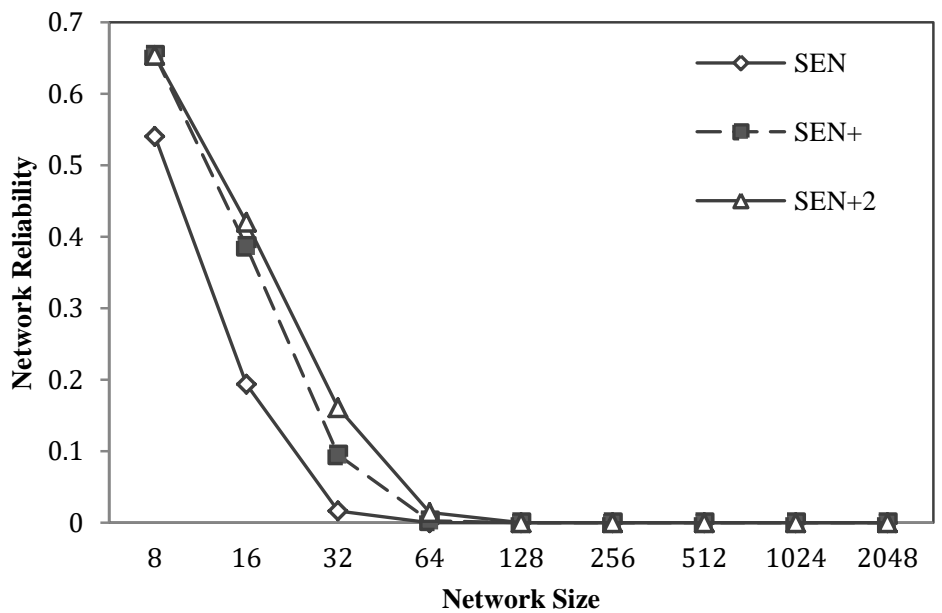

Figure 4. Time-independent network reliability vs. network size.

\section{Time-dependent reliability analysis}

One of the most important metrics that affects the reliability of a system is the time. Therefore, it is essential to consider this important parameter in the reliability analysis. However, so far, there has been no complete analysis of shuffle-exchange networks reliability as time-dependent. Another important point is that the terminal nodes such as processors (sources) and memory modules (destinations) are prone to failure in the network, which affects the reliability of the network. However, so far, in the time-dependent reliability analysis of MINs, the probability of failure has been merely intended for switch nodes. Therefore, in this section, the contribution is to fill these gaps. The assumptions made in this time-dependent analysis are listed as follows:

(1) Existing at least one fault-free path between each source-destination pair is necessary to deliver data between them. Therefore, in INs, reliability is defined as the probability of existence of at least one fault-free path between certain sets of sources and destinations.

(2) It will be assumed that each processor (source), memory module (destination), and switching element may fail.

(3) All failures are statistically independent.

(4) The failures are assumed to be exponentially distributed. Therefore, we define: $\gamma, \delta$, and $\lambda$ as the failure rate of a processor, a memory, and a $2 \times 2$ switching element, respectively. Then the corresponding reliabilities are given by: $R_{P}(t)=$ $\mathrm{e}^{-\gamma \mathrm{t}}, \mathrm{R}_{\mathrm{M}}(\mathrm{t})=\mathrm{e}^{-\delta \mathrm{t}}$, and $\mathrm{R}_{\mathrm{S}}(\mathrm{t})=\mathrm{e}^{-\lambda t}$. In addition, based on [32], it is assumed that a reasonable estimate for $\gamma$ and $\delta$ is equal to 0.0001 per hour. Also, according to research conducted on C.mmp system [30] (C.mmp is a canonical multiprocessor 
Fathollah Bistouni et al.

system with a $16 \times 16$ crossbar switch) and also based on [31], it is assumed that a reasonable estimate for $\lambda$ is equal to 0.0000017 per hour.

(5) The network components have two states: working or failing.

It should be noted that in order to obtain time-dependent reliability equations, time-dependent probabilities (i.e. component reliabilities) can be replaced with time-independent probabilities in time-independent reliability equations. In addition, the analysis carried out in this section has used a new methodology in comparison with previous works. In this analysis, in addition to the failure probability of the switches, the failure probability is considered for processors and memory modules as well. Furthermore, different failure rates are considered for each of the components.

Therefore, time-dependent terminal reliability, broadcast reliability, and network reliability for SEN, SEN+, and SEN+2 are given by:

$$
\begin{aligned}
& R_{T}(S E N)=e^{-\gamma t} e^{-\left(\log _{2} N\right) \lambda t} e^{-\delta t} \\
& R_{T}(S E N+)=e^{-\gamma t} e^{-\delta t}\left(e^{-2 \lambda t}\left(1-\left(1-e^{-\left(\left(\log _{2} N\right)-1\right) \lambda t}\right)^{2}\right)\right) \\
& R_{T}(\operatorname{SEN}+2)=e^{-\gamma t} e^{-\delta t}\left(e^{-2 \lambda t}\left(1-\left(1-\left(e^{-2 \lambda t}\left(1-\left(1-e^{-\left(\left(\log _{2} N\right)-2\right) \lambda t}\right)^{2}\right)\right)\right)^{2}\right)\right) \\
& R_{B}(S E N)=e^{-\gamma t} e^{-(N-1) \lambda t} e^{-N \delta t} \\
& R_{B}(S E N+)=e^{-\gamma t} e^{-N \delta t}\left(e^{-\left(\frac{(N+2)}{2}\right) \lambda t}\left(1-\left(1-e^{-\left(\frac{(N-2)}{2}\right) \lambda t}\right)^{2}\right)\right) \\
& R_{B}(S E N+2)=e^{-\gamma t} e^{-N \delta t}\left(\left(e^{-\left(\frac{(N+2)}{2}\right) \lambda t}\right)\left(1-\left(1-\left(e^{-2 \lambda t}\left(1-\left(1-e^{-\left(\frac{(N-4)}{4}\right) \lambda t}\right)^{2}\right)\right)\right)^{2}\right)\left(\left(1-\left(1-e^{-\lambda t}\right)^{2}\right)^{\frac{N-4}{4}}\right)\right) \\
& R_{N}(S E N)=e^{-N \gamma t} e^{-\left(\frac{N}{2} \log _{2} N\right) \lambda t} e^{-N \delta t} \\
& R_{N}(S E N+)=e^{-N \gamma t} e^{-N \delta t}\left(e^{-N \lambda t}\left(\left(1-\left(1-e^{-\lambda t}\right)^{2}\right)^{\frac{N}{4}}\right)\left(1-\left(1-e^{-\left(\frac{N}{4}\left(\left(\log _{2} N\right)-2\right)\right) \lambda t}\right)^{2}\right)\right) \\
& R_{N}(\operatorname{SEN}+2)=e^{-N \gamma t} e^{-N \delta t}\left(e^{-N \lambda t}\left(\left(1-\left(1-e^{-\lambda t}\right)^{2}\right)^{\frac{N}{4}}\right)\left(1-\left(1-e^{-\left(\frac{N}{8}\left(\left(\log _{2} N\right)-2\right)\right) \lambda t}\right)^{4}\right)\left(1-\left(1-e^{-\left(\frac{N}{4}\right) \lambda t}\right)^{2}\right)\right)
\end{aligned}
$$

According to Eqs. (10) through (12), the numerical results of time-dependent terminal reliability analysis for network sizes $(\mathrm{N})$ of 8,128 , and 512 , as a function of time are summarized in table 1 . First, consider the network size of 8 . According 
to table 1, for all operating times, both SEN+ and SEN+2 networks have a performance very close to each other in terms of terminal reliability. On the other hand, the terminal reliability of these networks is better than that of a SEN. This time, consider the network sizes of 128 and 512. According to table 1, SEN+ outperforms SEN in terms of terminal reliability for all operating times. In addition, SEN+2 outperforms SEN+ in terms of terminal reliability for all operating times. In other words, although the terminal reliability of SEN+ and SEN+2 are quite similar to each other in a small network size such as $8 \times 8$, SEN +2 outperforms SEN + in terms of terminal reliability in larger network sizes such as $128 \times 128$ and $512 \times 512$. As a result, for small-scale systems, adding one extra stage to SEN is a better option than two, in terms of terminal reliability. However, for systems with large sizes, adding two extra stages to SEN has a higher efficiency than one, in terms of terminal reliability.

Table 1. Terminal reliability as a function of time.

\begin{tabular}{|c|c|c|c|c|c|c|c|c|c|}
\hline \multirow{2}{*}{$\begin{array}{c}\text { Tim } \\
\text { e } \\
(\mathrm{Hr} \\
\text { ) }\end{array}$} & \multicolumn{3}{|c|}{$\mathbf{N}=\mathbf{8}$} & \multicolumn{3}{|c|}{$N=128$} & \multicolumn{3}{|c|}{$N=512$} \\
\hline & SEN & $\begin{array}{c}\text { SEN } \\
+\end{array}$ & $\begin{array}{c}\text { SEN } \\
+2\end{array}$ & SEN & $\begin{array}{c}\text { SEN } \\
+\end{array}$ & $\begin{array}{c}\text { SEN } \\
+2\end{array}$ & SEN & $\begin{array}{c}\text { SEN } \\
+\end{array}$ & $\begin{array}{c}\text { SEN } \\
+2\end{array}$ \\
\hline 500 & $\begin{array}{c}0.9025 \\
33\end{array}$ & $\begin{array}{c}0.9032 \\
98\end{array}$ & $\begin{array}{c}0.9032 \\
98\end{array}$ & $\begin{array}{c}0.8994 \\
70\end{array}$ & $\begin{array}{c}0.9032 \\
77\end{array}$ & $\begin{array}{c}0.9032 \\
98\end{array}$ & $\begin{array}{c}0.8979 \\
42\end{array}$ & $\begin{array}{c}0.9032 \\
59\end{array}$ & $\begin{array}{c}0.9032 \\
98\end{array}$ \\
\hline 1000 & $\begin{array}{c}0.8145 \\
66\end{array}$ & $\begin{array}{c}0.8159 \\
42\end{array}$ & $\begin{array}{c}0.8159 \\
42\end{array}$ & $\begin{array}{c}0.8090 \\
46\end{array}$ & $\begin{array}{c}0.8158 \\
68\end{array}$ & $\begin{array}{c}0.8159 \\
42\end{array}$ & $\begin{array}{c}0.8062 \\
99\end{array}$ & $\begin{array}{c}0.8158 \\
03\end{array}$ & $\begin{array}{c}0.8159 \\
42\end{array}$ \\
\hline 1500 & $\begin{array}{c}0.7351 \\
73 \\
\end{array}$ & $\begin{array}{c}0.7370 \\
31 \\
\end{array}$ & $\begin{array}{c}0.7370 \\
30 \\
\end{array}$ & $\begin{array}{c}0.7277 \\
12 \\
\end{array}$ & $\begin{array}{c}0.7368 \\
80 \\
\end{array}$ & $\begin{array}{c}0.7370 \\
29 \\
\end{array}$ & $\begin{array}{c}0.7240 \\
10 \\
\end{array}$ & $\begin{array}{c}0.7367 \\
49 \\
\end{array}$ & $\begin{array}{c}0.7370 \\
28 \\
\end{array}$ \\
\hline 2000 & $\begin{array}{c}0.6635 \\
17\end{array}$ & $\begin{array}{c}0.6657 \\
47\end{array}$ & $\begin{array}{c}0.6657 \\
47\end{array}$ & $\begin{array}{c}0.6545 \\
55\end{array}$ & $\begin{array}{c}0.6655 \\
06\end{array}$ & $\begin{array}{c}0.6657 \\
44\end{array}$ & $\begin{array}{c}0.6501 \\
19\end{array}$ & $\begin{array}{c}0.6652 \\
98\end{array}$ & $\begin{array}{c}0.6657 \\
42\end{array}$ \\
\hline 2500 & $\begin{array}{c}0.5988 \\
46\end{array}$ & $\begin{array}{c}0.6013 \\
54\end{array}$ & $\begin{array}{c}0.6013 \\
54\end{array}$ & $\begin{array}{c}0.5887 \\
52\end{array}$ & $\begin{array}{c}0.6010 \\
16\end{array}$ & $\begin{array}{c}0.6013 \\
49\end{array}$ & $\begin{array}{c}0.5837 \\
69\end{array}$ & $\begin{array}{c}0.6007 \\
25\end{array}$ & $\begin{array}{c}0.6013 \\
45\end{array}$ \\
\hline 3000 & $\begin{array}{c}0.5404 \\
79 \\
\end{array}$ & $\begin{array}{c}0.5431 \\
86\end{array}$ & $\begin{array}{c}0.5431 \\
86 \\
\end{array}$ & $\begin{array}{c}0.5295 \\
65\end{array}$ & $\begin{array}{c}0.5427 \\
49 \\
\end{array}$ & $\begin{array}{c}0.5431 \\
79 \\
\end{array}$ & $\begin{array}{c}0.5241 \\
91 \\
\end{array}$ & $\begin{array}{c}0.5423 \\
74 \\
\end{array}$ & $\begin{array}{c}0.5431 \\
72 \\
\end{array}$ \\
\hline 3500 & $\begin{array}{c}0.4878 \\
00\end{array}$ & $\begin{array}{c}0.4906 \\
42\end{array}$ & $\begin{array}{c}0.4906 \\
42\end{array}$ & $\begin{array}{c}0.4763 \\
27\end{array}$ & $\begin{array}{c}0.4901 \\
07\end{array}$ & $\begin{array}{c}0.4906 \\
32\end{array}$ & $\begin{array}{c}0.4706 \\
93\end{array}$ & $\begin{array}{c}0.4896 \\
51\end{array}$ & $\begin{array}{c}0.4906 \\
22\end{array}$ \\
\hline 4000 & $\begin{array}{c}0.4402 \\
55\end{array}$ & $\begin{array}{c}0.4431 \\
79\end{array}$ & $\begin{array}{c}0.4431 \\
78\end{array}$ & $\begin{array}{c}0.4284 \\
42\end{array}$ & $\begin{array}{c}0.4425 \\
51\end{array}$ & $\begin{array}{c}0.4431 \\
65\end{array}$ & $\begin{array}{c}0.4226 \\
55\end{array}$ & $\begin{array}{c}0.4420 \\
17\end{array}$ & $\begin{array}{c}0.4431 \\
51 \\
\end{array}$ \\
\hline
\end{tabular}

According to Eqs. (13) through (15), time-dependent broadcast reliability analysis for operating times from 500 to 4000 hours and network sizes (N) of 8, 128, and 512 are listed in table 2. According to this table, in all network sizes and at all operating times, SEN+2 has a better broadcast reliability compared to SEN and SEN+. Also, SEN+ outperforms SEN in terms of broadcast reliability.

Considering Eqs. (16) through (18), the results of time-dependent network reliability analysis versus time for small network size of 8 , middle network size of 128, and relatively large network size of 512 are shown in table 3. First, consider the network size of 8 . As table 3 shows, the best results belong to the SEN+ and SEN+2. Reliabilities of these networks are quite close to each other and are higher 
Fathollah Bistouni et al.

than the reliability of the SEN. Now, consider the network sizes of 128 and 512. As table 3 shows, SEN+2 is better than two other networks in terms of network reliability for all operating times.

In total, according to tables 1, 2, and 3, it can be concluded that for small network sizes such as $8 \times 8$, one additional stage has a better impact on the performance of MINs than two. However, for large-scale systems, the situation is vice versa. In other words, determining the number of stages to improve the reliability of the MINs efficiently needs to consider the size of the system.

Table 2. Broadcast reliability as a function of time.

\begin{tabular}{|c|c|c|c|c|c|c|c|c|c|}
\hline \multirow{2}{*}{$\begin{array}{c}\mathrm{Ti} \\
\mathrm{me} \\
(\mathrm{Hr} \\
)\end{array}$} & \multicolumn{3}{|c|}{$N=8$} & \multicolumn{3}{|c|}{$N=128$} & \multicolumn{3}{|c|}{$N=512$} \\
\hline & SEN & $\begin{array}{c}\text { SEN } \\
+\end{array}$ & $\begin{array}{c}\text { SEN } \\
+2\end{array}$ & SEN & SEN+ & $\begin{array}{c}\text { SEN+ } \\
2\end{array}$ & SEN & SEN+ & $\begin{array}{c}\text { SEN+ } \\
2\end{array}$ \\
\hline \multirow{2}{*}{500} & 0.633 & 0.634 & 0.634 & 0.0014 & 0.0014 & 0.0014 & 4.696 & 5.606 & 5.826 \\
\hline & 845 & 920 & 922 & 19 & 91 & 95 & E-12 & E-12 & E-12 \\
\hline 100 & 0.401 & 0.403 & 0.403 & 0.0000 & 0.0000 & 0.0000 & 2.205 & 2.976 & 3.389 \\
\hline 0 & 760 & 118 & 123 & 02 & 02 & 02 & E-23 & E-23 & E-23 \\
\hline 150 & 0.254 & 0.255 & 0.255 & 2.856 & 3.271 & 3.344 & 1.035 & 1.527 & 1.964 \\
\hline 0 & 654 & 941 & 948 & E-9 & E-9 & E-9 & E-34 & E-34 & E-34 \\
\hline 200 & 0.161 & 0.162 & 0.162 & 4.052 & 4.817 & 5.000 & 4.862 & 7.655 & 1.132 \\
\hline 0 & 411 & 496 & 503 & E-12 & E-12 & E-12 & E-46 & E-46 & E-45 \\
\hline 250 & 0.102 & 0.103 & 0.103 & 5.749 & 7.069 & 7.474 & 2.283 & 3.778 & 6.485 \\
\hline 0 & 310 & 167 & 174 & E-15 & E-15 & E-15 & E-57 & E-57 & E-57 \\
\hline 300 & 0.064 & 0.065 & 0.065 & 8.157 & 1.034 & 1.117 & 1.072 & 1.843 & 3.687 \\
\hline 0 & 849 & 498 & 505 & E-18 & E-17 & E-17 & E-68 & E-68 & E-68 \\
\hline 350 & 0.041 & 0.041 & 0.041 & 1.157 & 1.510 & 1.669 & 5.035 & 8.912 & 2.081 \\
\hline 0 & 104 & 583 & 589 & E-20 & E-20 & E-20 & E-80 & E-80 & E-79 \\
\hline 400 & 0.026 & 0.026 & 0.026 & 1.642 & 2.199 & 2.493 & 2.364 & 4.282 & 1.166 \\
\hline 0 & 054 & 400 & 404 & E-23 & E-23 & E-23 & E-91 & E-91 & E-90 \\
\hline
\end{tabular}

Table 3. Network reliability as a function of time.

\begin{tabular}{|c|c|c|c|c|c|c|c|c|c|}
\hline \multirow{2}{*}{$\begin{array}{c}\mathrm{Ti} \\
\mathrm{me} \\
(\mathrm{H} \\
\mathrm{r})\end{array}$} & \multicolumn{3}{|c|}{$\boldsymbol{N}=\mathbf{8}$} & \multicolumn{3}{|c|}{$N=128$} & \multicolumn{3}{|c|}{$N=512$} \\
\hline & SEN & $\begin{array}{c}\text { SEN } \\
+\end{array}$ & $\begin{array}{c}\text { SEN } \\
+2\end{array}$ & SEN & $\begin{array}{c}\text { SEN } \\
+\end{array}$ & $\begin{array}{c}\text { SEN } \\
+2\end{array}$ & SEN & SEN+ & $\begin{array}{c}\text { SEN+ } \\
2\end{array}$ \\
\hline \multirow{2}{*}{500} & 0.444 & 0.446 & 0.446 & 0.000 & 0.000 & 0.000 & $8.196 \mathrm{E}$ & $2.691 \mathrm{E}$ & $3.682 \mathrm{E}$ \\
\hline & 769 & 282 & 282 & 002 & 002 & 002 & -24 & -23 & -23 \\
\hline 100 & 0.197 & 0.199 & 0.199 & 3.559 & 5.783 & 6.112 & 6.717E & $5.489 \mathrm{E}$ & $1.249 \mathrm{E}$ \\
\hline 0 & 819 & 166 & 166 & E-12 & E-12 & E-12 & -47 & -46 & -45 \\
\hline 150 & 0.087 & 0.088 & 0.088 & 6.714 & 1.347 & 1.507 & $5.505 \mathrm{E}$ & $1.026 \mathrm{E}$ & $3.844 \mathrm{E}$ \\
\hline 0 & 984 & 883 & 883 & E-18 & E-17 & E-17 & -70 & -68 & -68 \\
\hline 200 & 0.039 & 0.039 & 0.039 & 1.266 & 3.096 & 3.706 & $4.512 \mathrm{E}$ & $1.851 \mathrm{E}$ & $1.093 \mathrm{E}$ \\
\hline 0 & 133 & 666 & 666 & E-23 & E-23 & E-23 & -93 & -91 & -90 \\
\hline 250 & 0.017 & 0.017 & 0.017 & 2.389 & 7.039 & 9.090 & $3.698 \mathrm{E}$ & $3.288 \mathrm{E}$ & $2.935 \mathrm{E}$ \\
\hline 0 & 405 & 701 & 701 & E-29 & E-29 & E-29 & -116 & -114 & -113 \\
\hline 300 & 0.007 & 0.007 & 0.007 & 4.507 & 1.587 & 2.223 & $3.031 \mathrm{E}$ & $5.801 \mathrm{E}$ & $7.565 \mathrm{E}$ \\
\hline 0 & 741 & 899 & 899 & E-35 & E-34 & E-34 & -139 & -137 & -136 \\
\hline
\end{tabular}




\begin{tabular}{cccccccccc}
\hline 350 & 0.003 & 0.003 & 0.003 & 8.503 & 3.552 & 5.416 & $2.484 \mathrm{E}$ & $1.020 \mathrm{E}$ & $1.895 \mathrm{E}$ \\
0 & 443 & 525 & 525 & $\mathrm{E}-41$ & $\mathrm{E}-40$ & $\mathrm{E}-40$ & -162 & -159 & -158 \\
\hline 400 & 0.001 & 0.001 & 0.001 & 1.604 & 7.907 & 1.315 & $2.036 \mathrm{E}$ & $1.790 \mathrm{E}$ & $4.649 \mathrm{E}$ \\
0 & 531 & 573 & 573 & $\mathrm{E}-46$ & $\mathrm{E}-46$ & $\mathrm{E}-45$ & -185 & -182 & -181 \\
\hline
\end{tabular}

\section{Mean time to failure (MTTF)}

Another important metric that we should investigate is the time that a system is available, often referred to as "uptime" in the IT industry. "Time to failure" of a system can be defined as the length of time that a system is online between outages or failures. The mean time to failure (MTTF) is the average of the time to failure or in other words, the MTTF is the expected value of the time to failure. Therefore, because of crucial nature of this parameter, it also will be analyzed in this paper.

The MTTF is calculated by the following equation:

MTTF $=\int_{0}^{\infty} R(t) d t$

According to Eq. (19), MTTF can be calculated by integrating the time-dependent reliability equation over time from the starting time at $t=0$ to infinity. Consequently, if the reliability equations obtained in the previous section are placed in Eq. (19), the MTTF equation can be obtained for each network. As an example, consider the terminal reliability of the SEN network (Eq. (10)). To obtain the MTTF equation for this reliability equation, Eq. (10) should be placed in Eq. (19). As a result, we have:

$$
\begin{aligned}
\operatorname{MTTF}_{T}(\text { SEN })= & \int_{0}^{\infty}\left(e^{-\gamma t} e^{-\left(\log _{2} N\right) \lambda t} e^{-\delta t}\right) d t \\
& =\left(\lim _{t \rightarrow \infty}-\frac{\ln (2) e^{-t \lambda l o g_{2} N-t \gamma-t \delta}}{\lambda \ln (N)+\ln (2) \gamma+\ln (2) \delta}\right)+\frac{\ln (2)}{\lambda \ln (N)+\ln (2) \gamma+\ln (2) \delta}
\end{aligned}
$$

In addition, in order to obtain the numerical results for Eq. (20), it is enough to place the appropriate values for different variables including the failure rates of the different nodes (i.e. $\lambda, \gamma$, and $\delta$ ) and the size of the network (i.e. $\mathrm{N}$ ).

Therefore, according to Eq. (19), we have:

$$
\begin{aligned}
& \operatorname{MTTF}_{T}(\operatorname{SEN}+)=\int_{0}^{\infty}\left(e^{-\gamma t} e^{-\delta t}\left(e^{-2 \lambda t}\left(1-\left(1-e^{-\left(\left(\log _{2} N\right)-1\right) \lambda t}\right)^{2}\right)\right)\right) d t \\
& \operatorname{MTTF}_{T}(\operatorname{SEN}+2)=\int_{0}^{\infty}\left(e^{-\gamma t} e^{-\delta t}\left(e^{-2 \lambda t}\left(1-\left(1-\left(e^{-2 \lambda t}\left(1-\left(1-e^{-\left(\left(\log _{2} N\right)-2\right) \lambda t}\right)^{2}\right)\right)\right)^{2}\right)\right)\right) d t
\end{aligned}
$$


Fathollah Bistouni et al.

$$
\begin{aligned}
& \operatorname{MTTF}_{B}(S E N)=\int_{0}^{\infty}\left(e^{-\gamma t} e^{-(N-1) \lambda t} e^{-N \delta t}\right) d t \\
& \operatorname{MTTF}_{B}(S E N+)=\int_{0}^{\infty}\left(e^{-\gamma t} e^{-N \delta t}\left(e^{-\left(\frac{(N+2)}{2}\right) \lambda t}\left(1-\left(1-e^{-\left(\frac{(N-2)}{2}\right) \lambda t}\right)^{2}\right)\right)\right) d t \\
& \operatorname{MTTF}_{B}(S E N+2)=\int_{0}^{\infty}\left(e ^ { - \gamma t } e ^ { - N \delta t } \left(( e ^ { - ( \frac { ( N + 2 ) } { 2 } ) \lambda t } ) ( 1 - ( 1 - ( e ^ { - 2 \lambda t } ( 1 - ( 1 - e ^ { - ( \frac { ( N - 4 ) } { 4 } ) \lambda t } ) ^ { 2 } ) ) ) ^ { 2 } ) \left(\left(1-\left(1-e^{\left.\left.\left.\left.-\lambda t)^{2}\right)^{\frac{N-4}{4}}\right)\right)\right)}\right) d t\right.\right.\right.\right. \\
& \operatorname{MTTF}_{N}(S E N)=\int_{0}^{\infty}\left(e^{-N \gamma t} e^{-\left(\frac{N}{2} \log _{2} N\right) \lambda t} e^{-N \delta t}\right) d t \\
& \operatorname{MTTF}_{N}(S E N+)=\int_{0}^{\infty}\left(e^{-N \gamma t} e^{-N \delta t}\left(e^{-N \lambda t}\left(\left(1-\left(1-e^{-\lambda t}\right)^{2}\right)^{\frac{N}{4}}\right)\left(1-\left(1-e^{-\left(\frac{N}{4}\left(\left(\log _{2} N\right)-2\right)\right) \lambda t}\right)^{2}\right)\right)\right) d t \\
& \operatorname{MTTF}_{N}(\operatorname{SSEN}+2)=\int_{0}^{\infty}\left(e^{-N \gamma t} e^{-N \delta t}\left(e^{-N \lambda t}\left(\left(1-\left(1-e^{-\lambda t}\right)^{2}\right)^{\frac{N}{4}}\right)\left(1-\left(1-e^{-\left(\frac{N}{8}\left(\left(\log _{2} N\right)-2\right)\right) \lambda t}\right)^{4}\right)\left(1-\left(1-e^{-\left(\frac{N}{4}\right) \lambda t}\right)^{2}\right)\right)\right) d t
\end{aligned}
$$

According to the Eqs. (20) through (22), terminal MTTF analysis results as a function of network size are summarized in table 4 . Considering this table, the best and worst results are obtained by the SEN+2 and SEN, respectively. In network size of 8, SEN+ and SEN+2 networks have a performance very close to each other in terms of terminal MTTF. However, as the network size increases, the advantage of $\mathrm{SEN}+2$ to SEN+ becomes more apparent. According to table 4, the reason is that although the terminal MTTF decreases by increasing network size in both SEN+ and SEN+2, the increase in network size has less negative impact on the reliability of the $\mathrm{SEN}+2$, compared to $\mathrm{SEN}+$. In other words, according to these results, in network size of 8 , it has been proved that annexation of one extra stage to the SEN is more rational than two, in terms of terminal MTTF. However, in network sizes of 16 onwards, adding two extra stages to the network is more efficient than one in terms of terminal MTTF.

Table 4. Terminal MTTF as a function of network size.

\begin{tabular}{cccc}
\hline $\begin{array}{c}\text { Time } \\
(\mathrm{Hr})\end{array}$ & SEN & SEN+ & SEN+2 \\
\hline 8 & 4875.6704046806435 & 4913.805954531999 & 4913.745085175846 \\
\hline 16 & 4835.5899419729203 & 4910.678199190802 & 4913.5651958112385 \\
\hline 32 & 4796.1630695443646 & 4906.452928482102 & 4913.264263108909 \\
\hline 64 & 4757.3739295908663 & 4901.209264894021 & 4912.834471130299 \\
\hline 128 & 4719.2071731949036 & 4895.02063456989 & 4912.264466541135 \\
\hline 256 & 4681.6479400749067 & 4887.955228061418 & 4911.540985544743 \\
\hline 512 & 4644.6818392940086 & 4880.076418732741 & 4910.650014367519 \\
\hline 1024 & 4608.294960875576 & 4871.443143228474 & 4909.577603338668 \\
\hline
\end{tabular}


Fathollah Bistouni et al.

$\begin{array}{llll}2048 & 4572.4737082761776 & 4862.11024776377 & 4908.310424454348\end{array}$

According to the Eqs. (23) through (25), broadcast MTTF analysis results as a function of network size are summarized in table 5. According to this table, SEN+ has higher broadcast MTTF compared to SEN, especially at small network sizes. However, as the network size increases, the performance of these two networks is close to each other in terms of broadcast MTTF. Also, SEN+2 achieves higher broadcast MTTF compared to SEN+. However, the superiority of SEN+2 compared to SEN+ is weak and they have a close performance, especially in large network sizes. In general, it can be concluded that the idea of increasing the number of stages in the MINs is not efficient idea in terms of broadcast MTTF, especially in large-scale systems. Although this idea leads to increase the hardware cost because of the increased number of switching elements, it does not provide a good performance in terms of broadcast MTTF, especially in large network sizes.

Table 5. Broadcast MTTF as a function of network size.

\begin{tabular}{ccccc}
\hline $\begin{array}{c}\text { Time } \\
(\mathrm{Hr})\end{array}$ & & SEN & SEN + & SEN+2 \\
\hline 8 & 1096.611470555 & 1100.6472445577 & 1100.6771433805 & 1096.6114705559 \\
& 9821 & 085 & 352 & 821 \\
\hline \multirow{2}{*}{16} & 579.5421616922 & 582.93342979215 & 582.98029851559 & 579.54216169226 \\
& 631 & 1 & 92 & 31 \\
\hline \multirow{2}{*}{32} & 298.2670683329 & 300.36507340000 & 300.39776433278 & 298.26706833298 \\
& 853 & 99 & 31 & 53 \\
\hline \multirow{2}{*}{64} & 151.3523330962 & 152.51046055148 & 152.52930100537 & 151.35233309621 \\
& 147 & 723 & 586 & 47 \\
\hline \multirow{2}{*}{128} & 76.24333823832 & 76.850832738411 & 76.860900797548 & 76.243338238321 \\
& 14 & 46 & 08 & 4 \\
\hline \multirow{2}{*}{256} & 38.26506208506 & 38.576066296611 & 38.581265392078 & 38.265062085063 \\
& 32 & 71 & 32 & 2 \\
\hline \multirow{2}{*}{512} & 19.16858192747 & 19.325917272586 & 19.328558481204 & 19.168581927477 \\
& 76 & 455 & 48 & 6 \\
\hline \multirow{2}{*}{1024} & 9.593329182619 & 9.6724575171799 & 9.6737885866464 & \multirow{2}{*}{9.5933291826196} \\
\hline \multirow{2}{*}{2048} & 4.798927343760 & 4.8386070376840 & 4.8392751948194 & \multirow{2}{*}{4.7989273437601} \\
\hline
\end{tabular}

According to the Eqs. (26) through (28), network MTTF analysis results as a function of network size are summarized in table 6 . According to table 6 , for the network MTTF, there is a similar situation to broadcast MTTF. This means that although increasing the number of stages can lead to an improvement in the network reliability; this improvement is slight, especially in networks with large sizes.

In general, according to table 4, 5, and 6, it can be concluded that the idea of adding extra stages to the MINs, can lead to suitable improvement in terminal MTTF. Also, 
Fathollah Bistouni et al.

in small network sizes, augmenting one additional stage to the SEN meets higher performance, but in large network sizes, adding two is more acceptable in terms of terminal MTTF. However, in case of broadcast and network MTTF, the idea of increasing the number of stages cannot provide the network with a significant improvement.

Table 6. Network MTTF as a function of network size.

\begin{tabular}{|c|c|c|c|c|}
\hline $\begin{array}{l}\text { Time } \\
\text { (Hr) }\end{array}$ & & SEN & SEN+ & $\mathrm{SEN}+2$ \\
\hline 8 & $\begin{array}{c}617.1315724512 \\
466\end{array}$ & $\begin{array}{c}619.72406470627 \\
17\end{array}$ & $\begin{array}{c}619.72406468814 \\
35\end{array}$ & $\begin{array}{c}617.13157245124 \\
66\end{array}$ \\
\hline 16 & $\begin{array}{c}307.2763028515 \\
241\end{array}$ & $\begin{array}{c}309.85458307302 \\
326\end{array}$ & $\begin{array}{c}309.86271685516 \\
897\end{array}$ & $\begin{array}{c}307.27630285152 \\
41\end{array}$ \\
\hline 32 & $\begin{array}{c}152.9987760097 \\
919\end{array}$ & $\begin{array}{c}154.92074686864 \\
638 \\
\end{array}$ & $\begin{array}{c}154.93152972097 \\
994\end{array}$ & $\begin{array}{c}152.99877600979 \\
19\end{array}$ \\
\hline 64 & $\begin{array}{c}76.18235007313 \\
51\end{array}$ & $\begin{array}{c}77.455758679390 \\
39\end{array}$ & $\begin{array}{c}77.465807627026 \\
73\end{array}$ & $\begin{array}{c}76.182350073135 \\
1\end{array}$ \\
\hline 128 & $\begin{array}{c}37.93396455450 \\
35\end{array}$ & $\begin{array}{c}38.724923350878 \\
76\end{array}$ & $\begin{array}{c}38.732914102158 \\
695\end{array}$ & $\begin{array}{c}37.933964554503 \\
5\end{array}$ \\
\hline 256 & $\begin{array}{c}18.88902321083 \\
17\end{array}$ & $\begin{array}{c}19.360668120720 \\
426\end{array}$ & $\begin{array}{c}19.366459354244 \\
235\end{array}$ & $\begin{array}{c}18.889023210831 \\
7\end{array}$ \\
\hline 512 & $\begin{array}{c}9.405851191909 \\
5\end{array}$ & $\begin{array}{c}9.6792830620109 \\
01 \\
\end{array}$ & $\begin{array}{c}9.6832300075887 \\
65 \\
\end{array}$ & 9.4058511919095 \\
\hline 1024 & $\begin{array}{c}4.683752997601 \\
9\end{array}$ & $\begin{array}{c}4.8390406172212 \\
15\end{array}$ & $\begin{array}{c}4.8416149971682 \\
39 \\
\end{array}$ & 4.6837529976019 \\
\hline 2048 & $\begin{array}{c}2.332368043945 \\
5\end{array}$ & $\begin{array}{c}2.4191828257507 \\
435\end{array}$ & $\begin{array}{c}2.4208073762861 \\
853\end{array}$ & 2.3323680439455 \\
\hline
\end{tabular}

\section{Availability}

In reliability engineering, a reasonable assumption is to consider reparability in the system performance analysis. On the other hand, the most common system performance metric used in repairable systems is availability [33, 34]. The dictionary defines available as "present or ready for immediate use”. This definition has direct applicability in the word of telecommunications. However, from a technical standpoint, availability is defined as the degree to which a system, subsystem or equipment lies in a specified operable and committable state at the start of a mission, when the mission is called for at an unknown, i.e. a random time [33]. Simply put, availability is the portion of time a system is in a functioning condition. This is often described as a mission capable rate.

Often when a component fails, the system is not ideal in terms of detection, isolation, and reconfiguration procedures. This defect is called imperfect coverage. Coverage is defined as the probability of successful reconfiguration of a system considering a component fault happens [31]. Thus, this important factor is used to analyze the availability in this section. In fact, using this important factor will bring 
analysis closer to practical scenarios. In practical scenarios, systems are not ideal and there are some failure probabilities in their various functions. When a failure occurs in one of the switches of a MIN, some mechanisms are needed to deal with it by performing some tasks, such as identifying the failure and identifying its location [35, 36]. However, any of these tasks may be confronted with a problem. For example, if the number of failures at a particular time exceeds a certain value, their identification may be disturbed [37]. As another example, when network size increases, the number of network components also increases and the amount of redundant switches in the network can also be increased, which makes it difficult to detect the failure of the switches [31, 38]. Therefore, The effect of this uncovered failure can spread throughout the network, and it may lead to the failure of the entire system, even with the presence of redundancies in the network [35]. This mode is called coverage failure state.

A Markov model is used to combine imperfect coverage and repair in an availability model as a usual approach. However, for fault tolerant MINs the construction of an overall Markov model and its solution is difficult due to the complex structure. Hierarchical composition approach can solve this problem. In this approach, each subsystem is modeled as a Markov chain in which the system availability is modeled as series independent Markov components.

Moreover, since the switches have high reliability, a logical assumption would be to rule out multiple repairs on the system.

For series systems consisting of $\mathrm{N}$ components, we will use the Markov state transition diagram in Fig. 5. In this figure, we assume each component $s$ time to failure is exponentially distributed with failure rate of $\lambda$. In this figure, in state 0 , the system is functioning with all components operational. Also, in state 1 , system is failed with the failure in one of the components. In this state, a failed component can be repaired at rate of $\mu$.

The transition matrix (A) for Fig. 5 is given by:

$\mathbb{A}=\left(\begin{array}{cc}-\mathrm{N} \lambda & \mathrm{N} \lambda \\ \mu & -\mu\end{array}\right)$

By applying Eq. (30) [33], we can solve the state equations to determine the probabilities of state occupation $\mathbb{P}=\left[\begin{array}{ll}\mathrm{P}_{0} & \mathrm{P}_{1}\end{array}\right]$.

P. $\mathbb{A}=\overline{0}$

$=\left[\begin{array}{ll}\mathrm{P}_{0} & \mathrm{P}_{1}\end{array}\right] \cdot\left(\begin{array}{cc}-\mathrm{N} \lambda & \mathrm{N} \lambda \\ \mu & -\mu\end{array}\right)$

The set of linear equations is thus:

$-P_{0} N \lambda+P_{1} \mu=0$ 


$$
\begin{aligned}
& \mathrm{P}_{0} \mathrm{~N} \lambda-\mathrm{P}_{1} \mu=0 \\
& \mathrm{P}_{0}+\mathrm{P}_{1}=1
\end{aligned}
$$

According to Eqs. (31) through (33), we have:

$\mathrm{P}_{0}=\frac{\mu}{\mathrm{N} \lambda+\mu}$

$\mathrm{P}_{1}=\frac{\mathrm{N} \lambda}{\mathrm{N} \lambda+\mu}$

We define the set of all possible system states as $\mathrm{S}=\left\{\mathrm{S}_{0}, \mathrm{~S}_{1}, \ldots, \mathrm{S}_{\mathrm{r}}\right\}$ and a set $\mathrm{W}$ as the subset of $S$ containing only those states where the system is working. Therefore, the availability of the system (A) is the sum of all probabilities in W:

$A=\sum_{i \in W} P_{j}=P_{0}$

Therefore, availability for a series system consisting of $\mathrm{N}$ components (shown in Fig. 5) is calculated by the following equation:

$\mathrm{A}=\frac{\mu}{\mathrm{N} \lambda+\mu}$

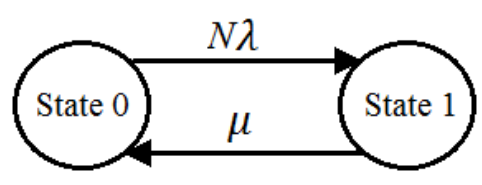

Figure 5. Series system Markov state transition diagram.

This time, consider a system consisting of two components in parallel. For this system, we will use the Markov state transition diagram in Fig. 6.

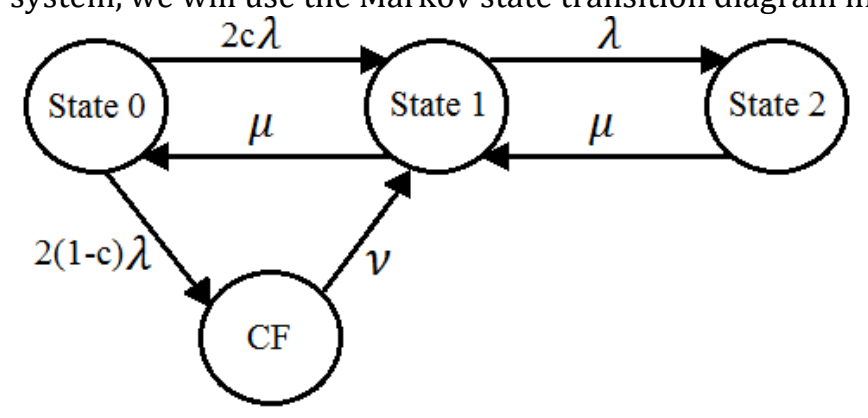

Figure 6. Parallel system Markov state transition diagram.

In Fig. 6, the system is functioning with both components operational in state 0; the 
$\mathbb{P} \cdot \mathbb{A}=\overline{0}$

system is functioning with only one component operational in state 1 ; and system is failed in state 2. In addition, in this figure, CF is the coverage failure state. If coverage is perfect $(c=1)$, system is operational as long as one of the two components is operational. However, if coverage is imperfect then there is probability $(1-c)$ that the system fails upon the failure of the first component (i.e. coverage failure state). Here it should be noted that, as shown in Fig. 6, there is a repair transition (with rate $v$ ) from the coverage failure state to state 1 . In this case, the main recovery method from coverage failure state is to reboot the system. Many researchers have developed this technique [39-42]. Generally, in this method, when a fault or failure is not detected due to imperfect coverage, it can be cleared by the reboot or reset operation. For this purpose, it is possible to consider a rate of reboot on a daily basis, for example, the rate proposed in [42] is 10 per day.

The transition matrix (A) for Fig. 6 is given by:

$$
\mathbb{A}=\left(\begin{array}{cccc}
-(2 c \lambda+2(1-c) \lambda) & 2 c \lambda & 0 & 2(1-c) \lambda \\
\mu & -(\mu+\lambda) & \lambda & 0 \\
0 & \mu & -\mu & 0 \\
0 & v & 0 & -v
\end{array}\right)
$$

By applying the state equation matrix definition to determine the linear algebraic equations in terms of state occupation probabilities, $\mathbb{P}=\left[\begin{array}{llll}\mathrm{P}_{0} & \mathrm{P}_{1} & \mathrm{P}_{2} & \mathrm{P}_{\mathrm{CF}}\end{array}\right]$.

$$
\begin{gathered}
=\left[\begin{array}{llll}
P_{0} & P_{1} & P_{2} & P_{C F}
\end{array}\right] \cdot\left(\begin{array}{cccc}
-(2 c \lambda+2(1-c) \lambda) & 2 c \lambda & 0 & 2(1-c) \lambda \\
\mu & -(\mu+\lambda) & \lambda & 0 \\
0 & \mu & -\mu & 0 \\
0 & v & 0 & -v
\end{array}\right) \\
-\mathrm{P}_{0}(2 c \lambda+2(1-c) \lambda)+\mathrm{P}_{1} \mu=0 \\
\\
2 \mathrm{P}_{0} c \lambda-\mathrm{P}_{1}(\mu+\lambda)+\mathrm{P}_{2} \mu+\mathrm{P}_{\mathrm{CF}} v=0 \\
\mathrm{P}_{1} \lambda-\mathrm{P}_{2} \mu=0 \\
2 \mathrm{P}_{0}(1-\mathrm{c}) \lambda-\mathrm{P}_{\mathrm{CF}} v=0 \\
\mathrm{P}_{0}+\mathrm{P}_{1}+\mathrm{P}_{2}+\mathrm{P}_{\mathrm{CF}}=1
\end{gathered}
$$

The availability of the system (A) is the sum of all probabilities in $\mathrm{W}$ :

$$
A=\sum_{i \in W} P_{j}=P_{0}+P_{1}
$$


Therefore, by solving the simultaneous equations for $\mathbb{P}$, we have:

$$
\begin{gathered}
A=\frac{v \mu^{2}}{v\left(\mu(2 \lambda+\mu)+2 \lambda^{2}\right)+2 \lambda(1-c) \mu^{2}}+\frac{\lambda \mu \nu}{v\left(\mu\left(\frac{\mu}{2}+\lambda\right)+\lambda^{2}\right)+\lambda(1-c) \mu^{2}} \\
=\frac{2 \lambda \mu \nu+v \mu^{2}}{v \mu^{2}+2 v \lambda^{2}+2 \lambda \mu \nu+2 \lambda \mu^{2}-2 c \lambda \mu^{2}}=\frac{1+2 \lambda / \mu}{1+2(\lambda / \mu)+2 \lambda(1-c) / v+2 \lambda^{2} / \mu^{2}}
\end{gathered}
$$

In parallel systems with more than two components, availability can be calculated by calculating the failure rates for the sub-systems and replacing this failure rate in the above equation with $\lambda$.

Now, given the above discussions, we will calculate the all-terminal availability for each of the networks of SEN, SEN+, and SEN+2. In this analysis, we will consider the following assumptions:

(1) It will be assumed that each switching element may fail.

(2) All failures are statistically independent.

(3) The failures are assumed to be exponentially distributed. Therefore, we define $\lambda$ as the failure rate of a $2 \times 2$ switching element (SE2). In addition, according to research conducted on C.mmp system [30] (C.mmp is a canonical multiprocessor system with a $16 \times 16$ crossbar switch) and also based on [31], it is assumed that a reasonable estimate for $\lambda$ is equal to 0.0000017 per hour.

(4) According to studies in [31], we will assume that $\mu=10^{4} \lambda$ and $\nu=\mu / 10$.

(5) The switches have high reliability. Therefore, a logical assumption would be to rule out multiple repairs on the system.

In terms of all-terminal reliability, the $\mathrm{N} \times \mathrm{N}$ SEN network is a system composed of $\mathrm{N} / 2\left(\log _{2} \mathrm{~N}\right)$ switching element in series. Therefore, availability for this network is calculated as follows:

$\mathrm{A}(\mathrm{SEN})=\frac{\mu}{\left(\frac{\mathrm{N}}{2}\left(\log _{2} \mathrm{~N}\right)\right) \lambda+\mu}$

In case of the SEN+ network, its RBD and Markov state transition diagram corresponding to each part of this RBD are shown in Fig. 7. 


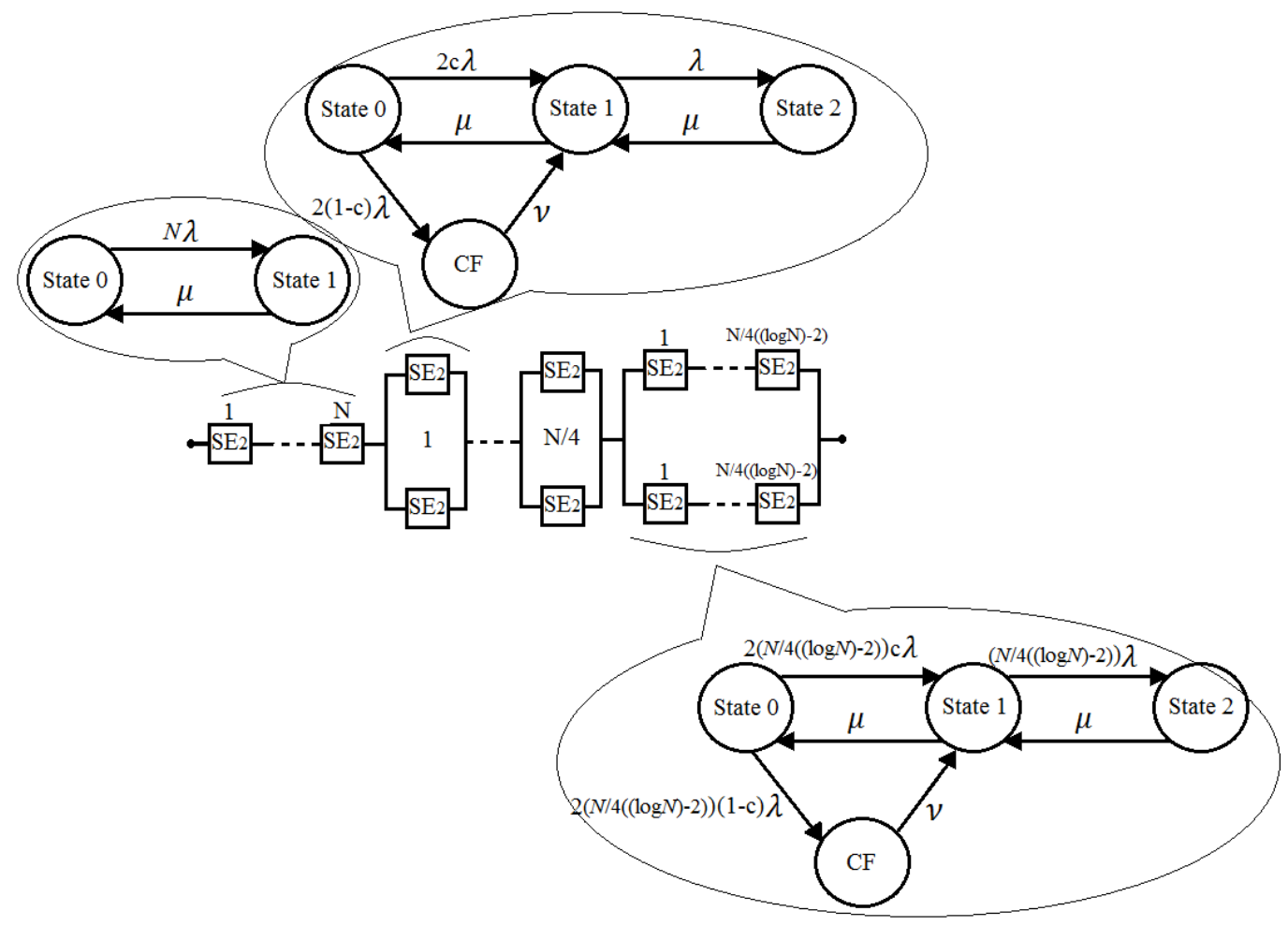

Figure 7. Markov state transition diagram for SEN+.

$A(S E N+)$

According to Fig. 7, availability for SEN+ network is given by:

$=\left(\frac{\mu}{N \lambda+\mu}\right)\left(\frac{1+2 \lambda / \mu}{1+2(\lambda / \mu)+2 \lambda(1-c) / v+2 \lambda^{2} / \mu^{2}}\right)^{\frac{N}{4}}\left(\frac{1+2\left(\frac{N}{4}\left(\left(\log _{2} N\right)-2\right)\right) \lambda / \mu}{\left.1+2\left(\left(\frac{N}{4}\left(\log _{2} N\right)-2\right)\right) \lambda / \mu\right)+2\left(\frac{N}{4}\left(\left(\log _{2} N\right)-2\right)\right) \lambda(1-c) / v+2\left(\left(\frac{N}{4}\left(\left(\log _{2} N\right)-2\right)\right) \lambda\right)^{2} / \mu^{2}}\right)$

Also, in case of the SEN+2 network, its RBD and Markov state transition diagram corresponding to each part of this RBD are shown in Fig. 8. According to this figure, availability for SEN+2 network is given by:

$$
\begin{aligned}
& A(S E N+2)=\left(\frac{\mu}{N \lambda+\mu}\right)\left(\frac{1+2 \lambda / \mu}{1+2(\lambda / \mu)+2 \lambda(1-c) / v+2 \lambda^{2} / \mu^{2}}\right)^{\frac{N}{4}}\left(\frac{1+2 \lambda_{S} / \mu}{1+2\left(\lambda_{S} / \mu\right)+2 \lambda_{S}(1-c) / v+2 \lambda_{S}{ }^{2} / \mu^{2}}\right) \\
& \left(\frac{1+2\left(\frac{N}{4}\right) \lambda / \mu}{1+2\left(\left(\frac{N}{4}\right) \lambda / \mu\right)+2\left(\frac{N}{4}\right) \lambda(1-c) / v+2\left(\left(\frac{N}{4}\right) \lambda\right)^{2} / \mu^{2}}\right)
\end{aligned}
$$

In Eq. (49), $\lambda_{S}$ is calculated as follows:

$$
\lambda_{S}=\frac{N \lambda\left(\log _{2}(N)-2\right)\left(1-e^{-\frac{N t \lambda\left(\log _{2}(N)-2\right)}{8}}\right)}{4\left(2-e^{-\frac{N t \lambda\left(\log _{2}(N)-2\right)}{8}}\right)}
$$




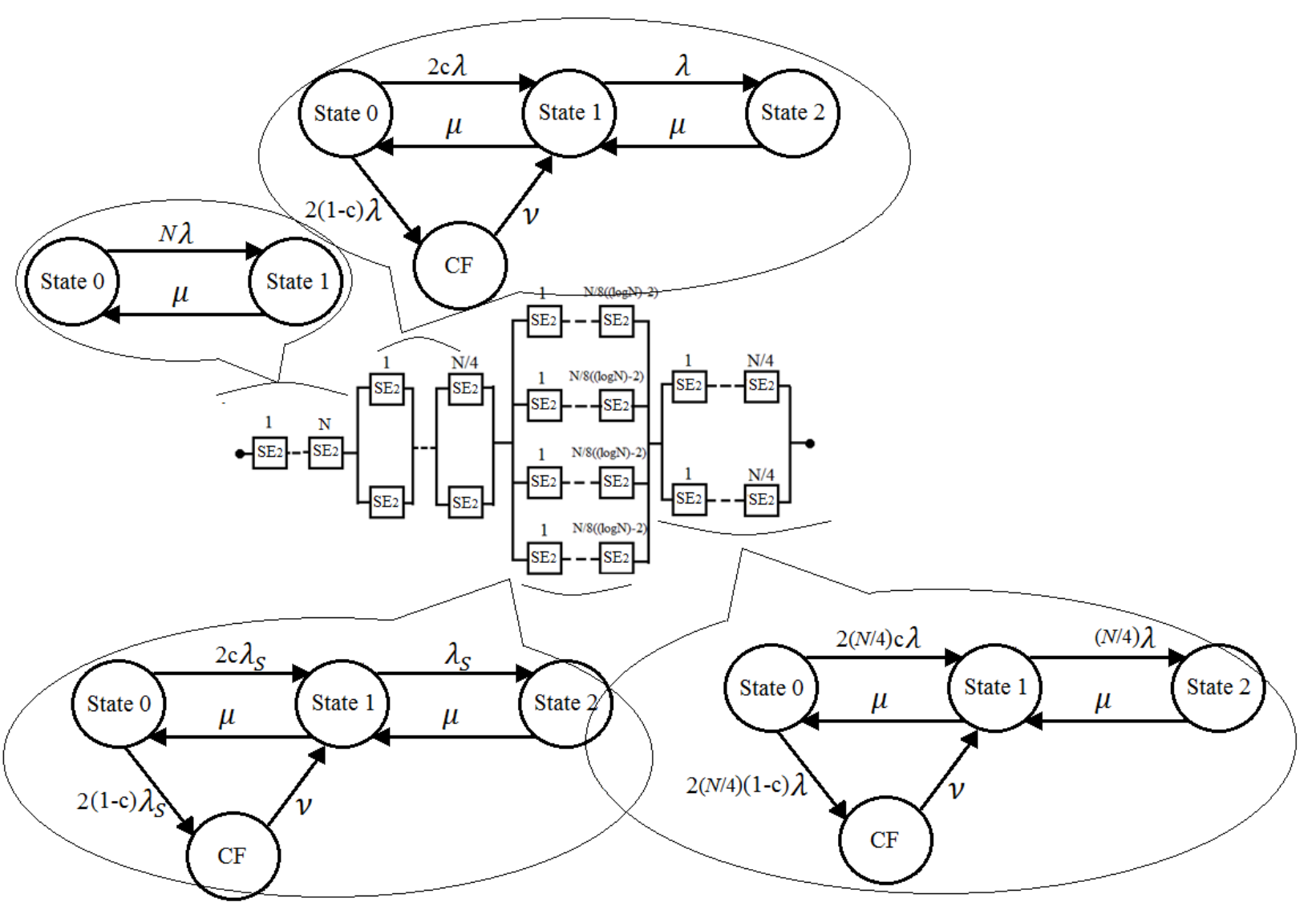

Figure 8. Markov state transition diagram for SEN+2.

According to Eqs. (47) through (50), the results of availability analysis as a function of network size are shown in Fig. 9. In these results, in the event of the SEN+ and $\mathrm{SEN}+2$, two cases have been considered: perfect coverage $(\mathrm{c}=1)$ and imperfect coverage ( $c=0.99$ ). According to this figure, the best and weakest results belong to SEN+2 ( $c=1)$ and SEN, respectively. Also, as the network size increases, SEN+2 achieves higher superiority compared to other networks. In other words, this result reflects the fact a MIN with two extra stages can provide higher efficiency than a MIN with one stage. However, for small-scale systems such as size $8 \times 8$ and $16 \times 16$, adding one stage is more efficient than two, in terms of availability due to cost-effectiveness reasons.

Another important point is the coverage parameter (c). As Fig. 9 shows, as long as this parameter is less than one, it has a significant negative impact on network availability. As a result, one way to improve availability of the networks is to use strategies to increase the probability of coverage. 


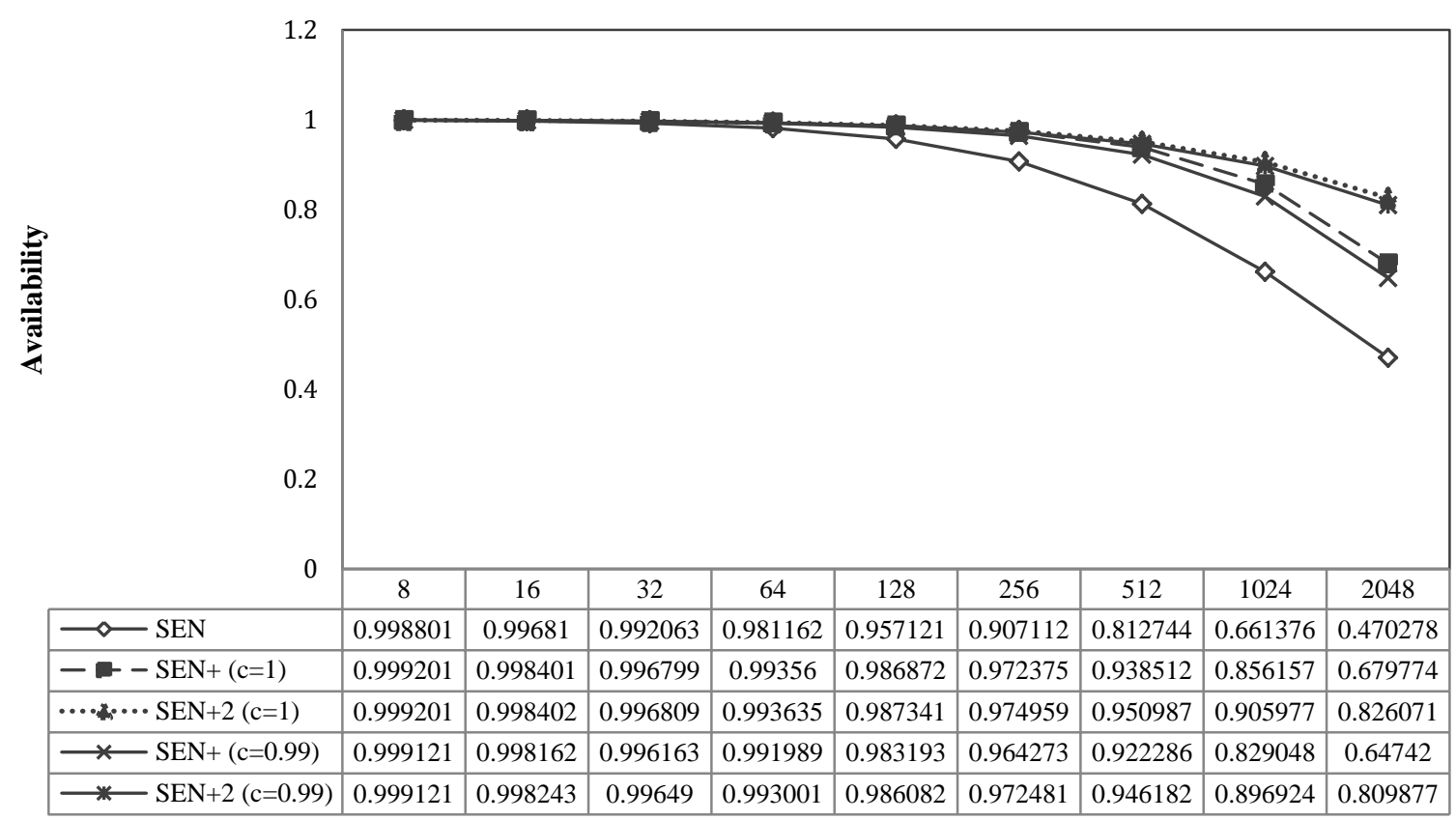

Figure 9. Availability as a function of network size.

\section{Discussion}

The parameters analyzed in this paper were as follows: (1) Time-independent terminal, broadcast, and network reliability analysis: In this case, the contribution of this paper was to analyze reliability of the MINs for different network sizes. The conclusion of this analysis indicates that as the network size increases, SEN+2 becomes more advantageous compared to two other networks in terms of reliability. (2) Time-dependent terminal reliability, broadcast reliability, and network reliability analysis: In this case, the contribution of this paper indicates that so far, there has been no complete analysis of shuffle-exchange networks reliability as time-dependent. Therefore, this is the first full time-dependent reliability analysis of SENs. Another important point is that processors (sources) and memory modules (destinations) are prone to failure in the network like switching elements. However, so far, the probability of failure has been merely intended for switch nodes. In contrast, this paper assumes that each processor (source), memory module (destination), and switching element may fail. The conclusion of this analysis is that as the network size increases, $\mathrm{SEN}+2$ gets a more appropriate choice compared to two other networks in terms of reliability. Conclusions derived from this analysis indicate that for small-scale systems, although adding one extra stage to SEN has lower hardware cost than two, it improves the reliability to a higher level. However, 
Fathollah Bistouni et al.

for large-scale systems, two extra stage MINs show greater competence compared to one, in terms of reliability. In other words, determining the number of stages to improve the reliability of the MINs efficiently needs to consider the size of the system. (3) Terminal MTTF, broadcast MTTF, and network MTTF analysis: In this case, the contribution of this paper was that there has been no complete analysis of shuffle-exchange networks MTTF. Therefore, this was the first full time-dependent MTTF analysis of SENs. Another important point is that in contrast to previous works, this paper assumes that each processor (source), memory module (destination) similarly to switching element may fail. Conclusions derived from this analysis are: The idea of adding extra stages to the MINs, can lead to suitable improvement in terminal MTTF. Also, in small network sizes, one extra stage SEN is more efficient, but in large network sizes, two extra stage SENs provide more benefits in terms of terminal MTTF. However, in case of broadcast and network MTTF, the idea of increasing the number of stages cannot provide a significant improvement to the network. (4) Availability analysis: In this case, the contribution of this paper is that almost none of the previous analyses on the reliability of the MINs have been discussed the reparability of system components in reliability analysis. Therefore, this paper analyzes the availability (most common system reliability metric used in repairable systems) of the SEN, SEN+, and SEN+2 with perfect and imperfect coverage as well as online-repair assumptions. Conclusions derived from this analysis are: To improve availability in systems with large sizes, concatenating two extra stages to a MIN is more suitable method. However, for small-scale systems such as size $8 \times 8$ and $16 \times 16$, using one extra stage provides more efficient condition for MINs than two in terms of availability due to cost-effectiveness reasons.

\section{Conclusion and future works}

In this paper, the impact of adding extra stages to MINs was extensively evaluated by reliability analyses on the three networks of SEN (one of the most popular MINs), SEN+ (SEN with one additional stage), and SEN+2 (SEN with two additional stages). In general, the results of this analysis are as follows: For small-scale systems, although adding one extra stage to SEN has lower hardware cost than two, it improves the reliability to a higher level. However, for large-scale systems, two extra stage MINs show greater competence compared to one, in terms of reliability, availability and terminal MTTF. However, in case of broadcast and network MTTF, the idea of increasing the number of stages cannot provide a significant improvement to the network. One of the most important future works in this domain is to calculate an optimal value for the number of switching stages 
added to various MINs in terms of reliability.

\section{Conflicts of Interest}

There is no conflict of interest

\section{References}

[1] Fathollah Bistouni and Mohsen Jahanshahi. "Determining the reliability importance of switching elements in the shuffle-exchange networks." International Journal of Parallel, Emergent and Distributed Systems (2018): 1-29.

[2] Fathollah Bistouni and Mohsen Jahanshahi. "Reliability analysis of multilayer multistage interconnection networks." Telecommunication Systems 62.3 (2016): 529-551.

[3] Neeraj Kumar Goyal and S. Rajkumar. "Multi-source multi-terminal reliability evaluation of interconnection networks." Microsystem Technologies 23.1 (2017): 255-274.

[4] Nima Jafarzadeh, et al. "Low Energy yet Reliable Data Communication Scheme for Network-on-Chip." IEEE Transactions on Computer-Aided Design of Integrated Circuits and Systems 34.12 (2015): 1892-1904.

[5] Mohsen Jahanshahi and Fathollah Bistouni. Crossbar-Based Interconnection Networks: Blocking, Scalability, and Reliability. Springer, Switzerland, 2018.

[6] Dimitris C. Vasiliadis, George E. Rizos, Costas Vassilakis, and E. Glavas. "Modelling and performance evaluation of a novel internal-priority routing scheme for finite-buffered multistage interconnection networks." International Journal of Parallel, Emergent and Distributed Systems 26.5 (2011): 381-397.

[7] John Garofalakis and Eleftherios Stergiou. "Analytical model for performance-evaluation of multistage interconnection networks supporting multi-class priority service." International Journal of Parallel, Emergent and Distributed Systems 26.5 (2011): 399-427.

[8] Nur Arzilawati Md Yunus and Mohamed Othman. "Empirical analysis of terminal reliability in multistage interconnection networks." Computational Intelligence and Efficiency in Engineering Systems. Springer International Publishing, 2015. 157-169.

[9] Nitin, Garhwal, Shruti, and Neha Srivastava. "Designing a fault-tolerant fully-chained combining switches multi-stage interconnection network with disjoint paths." Journal of Supercomputing 55.3 (2011): 400-431.

[10] Roselin Petagon and Jeeraporn Werapun. "Embedding the optimal all-to-all personalized exchange on multistage interconnection networks+." Journal of Parallel and Distributed Computing 88 (2016): 16-30.

[11] Mehrnaz Moudi and Mohamed Othman. "A GreedyZero algorithm to minimise the conflicts in an Optical Multistage Interconnection Network." Journal of Network and Computer Applications 41 (2014): 312-318.

[12] Dimitris C. Vasiliadis, George E. Rizos, and Costas Vassilakis. "Class-based weighted fair queuing scheduling on quad-priority Delta Networks." 
Fathollah Bistouni et al.

International Journal of Parallel, Emergent and Distributed Systems 27.5 (2012): 435-457.

[13] Dietmar Tutsch and Günter Hommel. "MLMIN: a multicore processor and parallel computer network topology for multicast." Computers \& Operations Research 35.12 (2008): 3807-3821.

[14] Eleftherios Stergiou and John Garofalakis. "Quantitative analysis of multilayer switching networks providing multiple internal routes." International Journal of Communication Systems (2018). https://doi.org/10.1002/dac.3785

[15] Indra Gunawan. "Redundant paths and reliability bounds in gamma networks." Applied Mathematical Modelling 32.4 (2008): 588-594.

[16] Indra Gunawan. "Reliability prediction of distributed systems using Monte Carlo method." International Journal of Reliability and Safety 7.3 (2013): 235-248.

[17] Fathollah Bistouni and Mohsen Jahanshahi. "Rearranging links: a cost-effective approach to improve the reliability of multistage interconnection networks." International Journal of Internet Technology and Secured Transactions 8.3 (2018): 336-373.

[18] Mohsen Jahanshahi and Fathollah Bistouni. "Improving the reliability of the Benes network for use in large-scale systems." Microelectronics Reliability 55.3 (2015): 679-695.

[19] Mohsen Jahanshahi and Fathollah Bistouni. "A new approach to improve reliability of the multistage interconnection networks." Computers \& Electrical Engineering 40.8 (2014): 348-374.

[20] Fathollah Bistouni and Mohsen Jahanshahi. "Improved extra group network: a new fault-tolerant multistage interconnection network." Journal of Supercomputing 69.1 (2014): 161-199.

[21] Fathollah Bistouni and Mohsen Jahanshahi. "Pars network: a multistage interconnection network with fault-tolerance capability." Journal of Parallel and Distributed Computing 75 (2015): 168-183.

[22] Fathollah Bistouni and Mohsen Jahanshahi. "Scalable crossbar network: a non-blocking interconnection network for large-scale systems." The Journal of Supercomputing 71.2 (2015): 697-728.

[23] S. Rajkumar and Neeraj Kumar Goyal. "Reliable multistage interconnection network design." Peer-to-Peer Networking and Applications 9.6 (2016): 979-990.

[24] Nur Arzilawati Md Yunus and Mohamed Othman. "Reliability Evaluation and Routing Integration in Shuffle Exchange Omega Network." Journal of Networks 9.7 (2014): 1732-1737.

[25] Fathollah Bistouni and Mohsen Jahanshahi. "Formulating broadcast reliability equations on multilayer multistage interconnection networks." The Journal of Supercomputing 71.11 (2015): 4019-4041.

[26] Fathollah Bistouni and Mohsen Jahanshahi. "Evaluating failure rate of fault-tolerant multistage interconnection networks using Weibull life distribution." Reliability Engineering \& System Safety 144 (2015): 128-146.

[27] James T. Blake and Kishor S. Trivedi. "Multistage interconnection network 
reliability." IEEE Transactions on Computers 38.11 (1989): 1600-1604.

[28] Indra Gunawan. "Reliability analysis of shuffle-exchange network systems." Reliability Engineering \& System Safety 93.2 (2008): 271-276.

[29] Fathollah Bistouni and Mohsen Jahanshahi. "Analyzing the reliability of shuffle-exchange networks using reliability block diagrams." Reliability Engineering \& System Safety 132 (2014): 97-106.

[30] Daniel P. Siewiorek, et al. "A case study of C.mmp, Cm*, and C.vmp: Part II-Predicting and calibrating reliability of multiprocessor systems." Proceedings of the IEEE 66.10 (1978): 1200-1220.

[31] James T. Blake and Kishor S. Trivedi. "Reliability analysis of interconnection networks using hierarchical composition." IEEE Transactions on Reliability 38.1 (1989): 111-120.

[32] Chita R. Das and Laxmi N. Bhuyan. "Bandwidth availability of multiple-bus multiprocessors." IEEE Transactions on Computers 100.10 (1985): 918-926.

[33] Mark L. Ayers. Telecommunications System Reliability Engineering, Theory, and Practice. John Wiley \& Sons, Hoboken, New Jersey. 2012.

[34] Fathollah Bistouni and Mohsen Jahanshahi. "Reliability Analysis of Ethernet Ring Mesh Networks." IEEE Transactions on Reliability 66.4 (2017): 1238-1252.

[35] Liudong Xing. "Reliability evaluation of phased-mission systems with imperfect fault coverage and common-cause failures." IEEE Transactions on Reliability 56.1 (2007): 58-68.

[36] S. Prabhudeva and A. K. Verma. "Coverage modeling and reliability analysis using multi-state function." International Journal of Automation and Computing 4.4 (2007): 380-387.

[37] Kuo-Hsiung Wang, Tseng-Chang Yen, and Jen-Ju Jian. "Reliability and sensitivity analysis of a repairable system with imperfect coverage under service pressure condition." Journal of Manufacturing Systems 32.2 (2013): 357-363.

[38] Madhu Jain. "Availability prediction of imperfect fault coverage system with reboot and common cause failure." International Journal of Operational Research 17.3 (2013): 374-397.

[39] Kishor S. Trivedi. Probability and Statistics with Reliability, Queueing, and Computer Science Applications. Wiley, New York, 2008.

[40] Jau-Chuan Ke, Ssu-Lang Lee, and Ying-Lin Hsu. "On a repairable system with detection, imperfect coverage and reboot: Bayesian approach." Simulation Modelling Practice and Theory 16.3 (2008): 353-367.

[41] Jau-Chuan Ke and Tzu-Hsin Liu. "A repairable system with imperfect coverage and reboot." Applied Mathematics and Computation 246 (2014): 148-158.

[42] Madhu Jain and Rakesh Kumar Meena. "Fault tolerant system with imperfect coverage, reboot and server vacation." Journal of Industrial Engineering International 13.2 (2017): 171-180. 